\title{
Quantum Glass of Interacting Bosons with Off-Diagonal Disorder Has a Severe Sign Problem
}

\author{
A.M. PieKarskA* And T.K. Kopé́ \\ Institute of Low Temperature and Structure Research, Polish Academy of Sciences, \\ P.O. Box 1410, 50-950 Wrocław 2, Poland
}

\begin{abstract}
We study a disordered system of interacting bosons, described by the Bose-Hubbard model with the disorder introduced via Gaussian-distributed hopping integrals. After applying the replica trick and the Trotter formula, we arrive at an expression for order parameters involving averaging with respect to the effective Hamiltonian, that has to be numerically evaluated. We show that the Monte Carlo algorithm fails because of the sign problem, which is severe in this case, hence cannot be removed via standard methods. It cannot be therefore excluded that this problem belongs to the NP-complete complexity class, i.e., of problems without known polynomial-time solution but with a possibility of validating a given solution candidate at such a computational complexity.
\end{abstract}

DOI: $10.12693 /$ APhysPolA.135.78

PACS/topics: 02.70.Tt, 05.30.Jp, 75.10.Nr, 67.85.Hj, 05.30.Rt

\section{Introduction}

Disordered systems are of great interest from the point of view of both condensed matter and statistical physics. The Anderson localization [1] is an example of a unique phenomenon that originates exclusively from disorder. In most cases, many-body systems pose a difficult challenge to be properly described theoretically, since the standard methods from single-particle systems break down when applied due to the exponential increase of the number of states to consider, which causes the solution to be unfeasible. Therefore, one needs to refrain to more complicated methods, like Green's functions [2] or density matrix renormalization group (DMRG) [3]. Theoretical description of randomness in such strongly correlated systems is an additional challenge to cope with beyond these usual difficulties found in interacting many-body systems. Many of the latter have been proven $[4,5]$ to belong to the NP-complete computational complexity class [6]. NP stands here for nondeterministic polynomial time and means that for a computational problem belonging to this class, the correctness of a given (e.g. guessed) solution can be numerically checked in time polynomially scaling with the system size. However, direct solving the problem is a more difficult task, and no algorithm of polynomial complexity that computes the result has yet been found. It may be shown that obtaining one will be equivalent to a discovery of a polynomial algorithm solving other problems belonging to this class (NP-complete problems). The existence of the latter is, however, uncertain.

The Monte Carlo method (MC) [7] is one of the most extensively used ways of simulating physical many-body and complex systems. Although in its standard version

*corresponding author; e-mail: a.piekarska@int.pan.wroc.pl suitable for classical physical systems, the error of the result is proven to be polynomially bounded, the effective problem emerging from the mapping of a quantum system to a classical one [8] may be impossible to be solved within such complexity. It has been shown [4] that the fermionic Ising spin problem is NP-complete and suffers from the unresolvable sign problem, which means that the error of the result obtained via MC grows exponentially with the system size. The hardness originates there from negative weights of some configurations, which upon their summation, leads to the partition function orders of magnitude smaller than its standard deviation.

In this work, we study a quantum many-body system of interacting bosons in which an off-diagonal disorder is introduced, i.e. it is incorporated into the kinetic energy term describing the hopping of particles between sites. This causes appearance of frustration in the system and hence a possible emergence of the glassy phase [9], which in competition with quantum fluctuations leads to interesting phenomena known from the field of quantum spin glasses [10], which has been recently shown [11].

Here, we focus on the computational complexity of the problem. It has been shown [5] that some of the Ising spin glass models are NP-complete, while some other are polynomially bounded. The glassy system investigated by us exhibits features analogous to those of spin glasses, hence we are interested in establishing whether it is possible to get rid of the sign problem of the bosonic glass. We show that the approach of average sign [12] fails in a way described by Kieu and Griffin [13]. As a result, we arrive at the conclusion that statistical averages in the system cannot be computed using this standard transformation. However, we do not establish whether it can be computed in polynomial time using another method.

\section{Model}

We study a system of bosons featuring off-diagonal disorder realized by a set of hopping integrals being 
independent random variables $J_{i j}$ of the Gaussian distribution with zero mean and variance equal to $J^{2} / N$. We also include the on-site interaction of strength $U$ and the chemical potential $\mu$. Hence, the Bose-Hubbard Hamiltonian describing said system has the following form:

$$
\begin{aligned}
\widehat{H} & =-\sum_{i<j} J_{i j}\left(\hat{a}_{i}^{\dagger} \hat{a}_{j}+\hat{a}_{j}^{\dagger} \hat{a}_{i}\right) \\
& +\frac{U}{2} \sum_{i} \hat{n}_{i}\left(\hat{n}_{i}-1\right)-\mu \sum_{i} \hat{n}_{i},
\end{aligned}
$$

where $\hat{a}_{i}\left(\hat{a}_{i}^{\dagger}\right)$ annihilates (creates) a particle at site $i$ $(1 \ldots N)$, and $\hat{n}_{i}=\hat{a}_{i}^{\dagger} \hat{a}_{i}$ is the particle number operator for site $i$.

We follow closely the quantum extension [14] of the method presented first by Sherrington and Kirkpatrick [15]. The resulting procedure includes employing the so-called replica trick [16] to simplify the expression for the free energy averaged over the $J_{i j}$ distributions. Then, using the Trotter expansion formula [17], one separates the non-commuting terms of the Hamiltonian, which is then followed by applying the HubbardStratonovich transformation to get rid of the higher order terms. The latter is done at the cost of introducing a set of new variables and finally one uses the saddle point method to find the values of these variables that minimize the free energy. Having this done, we arrive at the following system of self-consistent equations for variables $\lambda_{k k^{\prime}}$ that have been introduced via the Hubbard-Stratonovich transformation and serve as the order parameters of the effective system,

$$
\lambda_{k k^{\prime}}=\frac{J \beta}{2 M}\left\langle p_{k} p_{k^{\prime}}\right\rangle_{\mathrm{eff}},
$$

where $M$ denotes the size of the Trotter expansion that was carried out to obtain the final equations, $p_{k}$ and $q_{k}$ are the eigenvalues of the operators $\widehat{P}=\mathrm{i}\left(\hat{a}^{\dagger}-\hat{a}\right) / \sqrt{2}$ and $\widehat{Q}=\left(\hat{a}^{\dagger}+\hat{a}\right) / \sqrt{2}$, respectively, and $k$ is the Trotter index. The statistical averages are computed as follows:

$$
\langle\cdots\rangle_{\text {eff }}=\frac{\operatorname{Tr}_{p q} \cdots \mathrm{e}^{-\beta \widehat{H}_{\text {eff }}}}{\operatorname{Tr}_{p q} \mathrm{e}^{-\beta \widehat{H}_{\mathrm{eff}}}},
$$

and pose the main problem that is investigated in this contribution. The trace here means a summation over all possible configurations of $\left\{p_{1}, p_{2}, \ldots, p_{M} ; q_{1}, q_{2}, \ldots, q_{M}\right\}$. Since the operators $\widehat{P}$ and $\widehat{Q}$ have infinite spectra, the Hilbert space needs to be truncated, which is done via expressing the problem in the particle number eigenstates and limiting the number of particles to maximally a few. The average is then taken with respect to the effective Hamiltonian given by

$$
\begin{aligned}
& -\beta \widehat{H}_{\mathrm{eff}}=-\frac{1}{N}\left[\frac{J \beta}{2 M} \sum_{k}\left(p_{k}^{2}+q_{k}^{2}\right)\right]^{2} \\
& +\frac{J \beta}{M} \sum_{k, k^{\prime}} \lambda_{k k^{\prime}}\left(p_{k} p_{k^{\prime}}+q_{k} q_{k^{\prime}}\right)+\ln \mathcal{M}(p, q)
\end{aligned}
$$

$$
\mathcal{M}(p, q)=\prod_{k=1}^{M}\left\langle p_{k} \mid q_{k}\right\rangle\left\langle q_{k}\left|\mathrm{e}^{-\beta \widehat{H}_{U} / M}\right| p_{k+1}\right\rangle,
$$

where Hamiltonian $\widehat{H}_{U}=(U / 2) \hat{n}^{2}-(\mu+U / 2) \hat{n}$ is the interaction part of the original Hamiltonian. Finally, the critical lines that one wants to find in order to obtain the phase diagram of the system are given by a condition

$$
\sum_{k} \lambda_{k k^{\prime}}=\frac{1}{2}
$$

Note that the original size of the system $N$, has been integrated out, but a new size of the classical counterpart $M$, is introduced. The quantum-classical mapping performed here is exact in the limit of $M \rightarrow \infty$. However, the number of configurations increases exponentially with $M$, so calculations are achievable in reasonable time only for the lowest values of $M$.

\section{Discussion}

In the self-consistent Eqs. (2), we would like to compute the statistical averages according to the recipe from Eq. (3) in a computationally effective manner. The MC method, if applicable, would allow to obtain the critical lines for $M$ up to a few hundreds with use of a typical modern computer. However, the straightforward application of MC needs the weights to be real and positive, which is not satisfied in the studied equations, since $\mathcal{M}(p, q)$ may be complex. We would like to check if it is possible to modify the problem in a way that would allow the usage of MC.

First, one may notice that each summand

$$
s(p, q)=p_{k} p_{k^{\prime}} \exp \left(-\beta H_{\mathrm{eff}}(p, q)\right)
$$

is a complex conjugate of another one

$$
s(p, q)=s(-p, q)^{*} \Rightarrow s(p, q)+s(-p, q) \in \mathbb{R},
$$

and the only unmatched summands are real

$$
s(0, q) \in \mathbb{R},
$$

which allows us to drop the imaginary part of all summands without changing the final result. This leaves us with only real weights, but they might be negative so the sign problem still occurs.

We then check if the problem can be solved using a standard transformation [12]. To do this, we break up the weights $\exp \left(-\beta H_{\text {eff }}\right)$ into their sign and magnitude $\operatorname{sgn}\left(\exp \left(-\beta H_{\text {eff }}\right)\right)\left|\exp \left(-\beta H_{\text {eff }}\right)\right|$ and estimate the partition function and its variation [13] at the critical point. Let

$$
Z_{\text {eff }}=\operatorname{Tr}_{p q} \mathrm{e}^{-\beta \widehat{H}_{\text {eff }}}
$$

and

$$
\widetilde{Z}_{\text {eff }}=\operatorname{Tr}_{p q}\left|\mathrm{e}^{-\beta \widehat{H}_{\text {eff }}}\right| \text {. }
$$

We define new weights as:

$$
w(p, q)=\frac{\left|\mathrm{e}^{-\beta \widehat{H}_{\mathrm{eff}}}\right|}{\widetilde{Z}_{\mathrm{eff}}},
$$

and as a result the averages we desire to compute via $\mathrm{MC}$ method transform into 


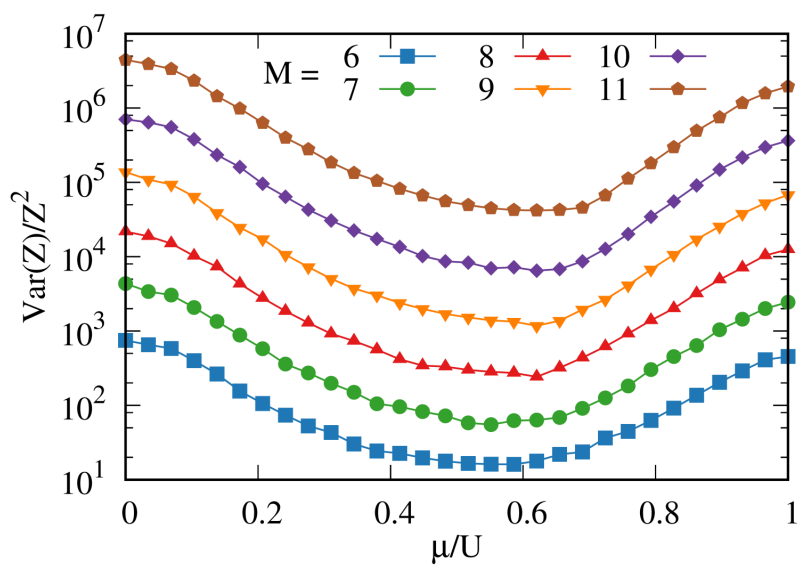

Fig. 1. The relative error of the partition function $Z$ of the system as a function of reduced chemical potential $\mu / U$ (colored symbols) for various values of the Trotter size $M$. Lines are to guide an eye only.

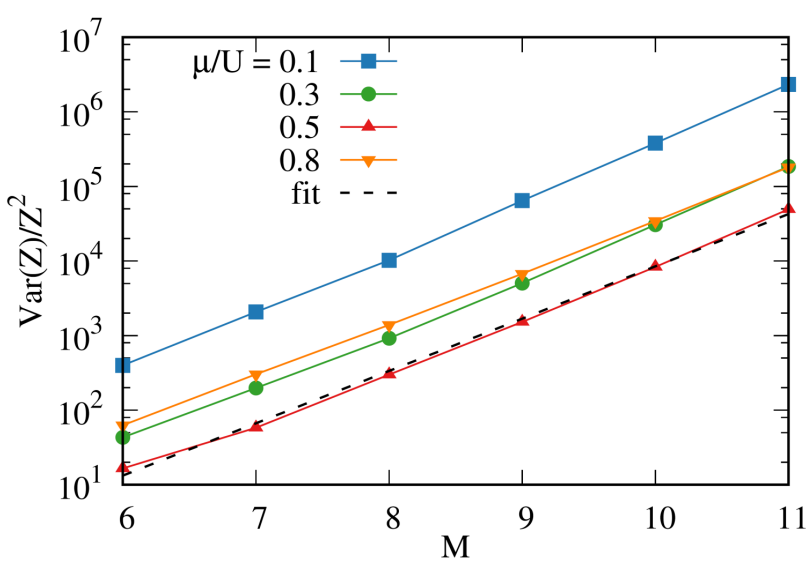

Fig. 2. The relative error of the partition function $Z$ of the system as a function of the Trotter size $M$ (colored symbols) for various values of the reduced chemical potential $\mu / U$. Solid lines are to guide an eye only. The data for $\mu / U=0.5$ is accompanied with a sample exponential fit (dashed black line) given by formula $\operatorname{Var}\left(Z_{\text {eff }}\right) / Z_{\text {eff }}^{2}=\exp (1.61493 M-7.10402)$.

$$
\langle\cdots\rangle_{\text {eff }}=\frac{\left\langle\left\langle\cdots \mathrm{e}^{-\beta \widehat{H}_{\mathrm{eff}}} / w(p, q)\right\rangle\right\rangle}{\left\langle\left\langle\mathrm{e}^{-\beta \widehat{H}_{\text {eff }}} / w(p, q)\right\rangle\right\rangle},
$$

where

$$
\langle\langle\cdots\rangle\rangle=\operatorname{Tr}_{p q} \cdots w(p, q)
$$

is the average weighted by the new set of weights. The latter are real and positive, which is a mandatory step towards applying the MC method. We then calculate the variance of the denominator

$$
\begin{gathered}
\operatorname{Var}\left(Z_{\text {eff }}\right)=\operatorname{Tr}_{p q}\left[\frac{\mathrm{e}^{-\beta \widehat{H}_{\text {eff }}}}{w(p, q)}-Z_{\text {eff }}\right]^{2} w(p, q)= \\
\widetilde{Z}_{\text {eff }}^{2} \operatorname{Tr}_{p q} \operatorname{sgn}^{2}\left(\mathrm{e}^{-\beta \widehat{H}_{\text {eff }}}\right)-Z_{\text {eff }}^{2}=\widetilde{Z}_{\text {eff }}^{2}-Z_{\text {eff }}^{2} .
\end{gathered}
$$

In case the error of $Z_{\text {eff }}$ is too big, the MC method fails despite the real weights. The value of $\operatorname{Var}\left(Z_{\text {eff }}\right) / Z_{\text {eff }}^{2}$ at the critical point plotted against the chemical potential is presented in Fig. 1 for various values of the Trotter size $M$. One may notice that the error exceeds the value by at least 2 orders of magnitude already for $M=7$ and the ratio increases with $M$. We investigate the dependence of the error on $M$ at several values of $\mu / U$ and present the corresponding results in Fig. 2. Fitting to these data reveals an exponential growth with respect to $M$. A sample fit for $\mu / U=0.5$ yields the dependency of $\operatorname{Var}\left(Z_{\text {eff }}\right) / Z_{\text {eff }}^{2}=\exp (1.61493 M-7.10402)$. Hence, we infer that MC calculation of this problem would be highly inaccurate due to the fluctuations of denominator, that are divergent with the system size.

\section{Conclusions}

We studied a quantum many-body system of interacting bosons. We took into account an off-diagonal disorder in the form of Gaussian-distributed hopping integrals, as well as on-site interactions and the chemical potential. On the way of solving the problem, we used the replica method and Trotter expansion formula. We arrived at the conclusion that the effective classical problem, resulting from the mapping of discussed quantum system of bosonic glass with off-diagonal disorder is impossible to solve using a modification of the $\mathrm{MC}$ algorithm. This resulted from the presence of the severe sign problem in the calculation of statistical averages. We estimated the error of the denominator in the expression for the statistical sum to be at least 2 orders of magnitude bigger than its value already for $M=7$ and divergent with $M$ exponentially, which makes the method unstable. We do not, however, make any claims about complexity class of this problem. Had the error scaled polynomially, we would conclude the problem to be polynomially solved based on [4]. Our opposite result does not settle the issue though. It would require making a link to some other problem already established in a certain complexity class. Examples of such proofs can be found in Refs. [4, 5].

\section{Acknowledgments}

Calculations have been carried out using resources provided by Wrocław Centre for Networking and Supercomputing (http://wcss.pl), grant No. 449.

\section{References}

[1] P.W. Anderson, Phys. Rev. 109, 1492 (1958).

[2] G. Green, An Essay on the Application of Mathematical Analysis to the Theories of Electricity and Magnetism, printed for the author by T. Wheelhouse, 1828 (facsimile Mayer and Müller, 1889).

[3] S.R. White, Phys. Rev. B 48, 10345 (1993).

[4] M. Troyer, U.-J. Wiese, Phys. Rev. Lett. 94, 170201 (2005).

[5] F. Barahona, J. Phys. A 15, 3241 (1982).

[6] C.H. Papadimitriou, in: Encyclopedia of Computer Science, Wiley, Chichester (UK) 2003, p. 260.

[7] N. Metropolis, S. Ulam, J. Am. Stat. Assoc. 44, 335 (1949). 
[8] A.W. Sandvik, J. Kurkijärvi, Phys. Rev. B 43, 5950 (1991).

[9] G. Toulouse, in: World Scientific Lecture Notes in Physics, World Sci., 1986, p. 99.

[10] A.J. Bray, M.A. Moore, J. Phys. C 13, L655 (1980).

[11] A.M. Piekarska, T.K. Kopeć, Phys. Rev. Lett. 120, 160401 (2018).

[12] H.D. Raedt, A. Lagendijk, Phys. Rev. Lett. 46, 77 (1981).
[13] T.D. Kieu, C.J. Griffin, Phys. Rev. E 49, 3855 (1994).

[14] K. Usadel, Nucl. Phys. B 5, 91 (1988).

[15] D. Sherrington, S. Kirkpatrick, Phys. Rev. Lett. 35, 1792 (1975).

[16] M. Mezard, G. Parisi, M. Virasoro, Spin Glass Theory and Beyond, World Sci., 1986.

[17] H.F. Trotter, Proc. Am. Math. Soc. 10, 545 (1959). 